\title{
EFECTO DE UN PROGRAMA DE PAUSA ACTIVA MÁS FOLLETOS INFORMATIVOS EN LA DISMINUCIÓN DE MOLESTIAS MUSCULOESQUELÉTICAS EN TRABAJADORES ADMINISTRATIVOS
}

\author{
V. Steffany Cáceres-Muñoz ${ }^{1, a}$, Antonella Magallanes-Meneses ${ }^{1, a}$, Dafne Torres-Coronel ${ }^{1, a}$, \\ Peter Copara-Moreno1,a, d, Manuel Escobar-Galindo ${ }^{1, b, c}$, Percy Mayta-Tristán ${ }^{1,2, e}$
}

\begin{abstract}
RESUMEN
Objetivos. Comprobar el efecto de un programa de pausa activa más folletos informativos, en la disminución de molestias musculoesqueléticas en trabajadores administrativos de una institución pública de Lima, Perú. Materiales y métodos. Se realizó un estudio cuasiexperimental con grupo control, no aleatorizado, en dos sedes en una institución pública. El grupo intervención $(n=135)$ recibió folletos informativos una vez por semana, adicionalmente realizó el programa de pausas activas supervisadas, las cuales consistían en ejercicios de respiración, estiramiento y calentamiento. El grupo control $(n=127)$ solo recibió folletos informativos una vez por semana. Se midió la frecuencia e intensidad de molestias musculoesqueléticas en los últimos siete días. Resultados. La edad promedio fue 38 años. Los segmentos más afectados fueron el cuello y dorso lumbar. El grupo intervención redujo $20 \%$ la frecuencia de molestias musculoesqueléticas en cuello en los últimos siete días $(p<0,001)$ y $17 \%$ en la zona dorso lumbar $(p<0,001)$, el grupo control solo redujo la frecuencia en la zona dorso lumbar $(p=0,029)$. Respecto a intensidad del dolor, el grupo de intervención tuvo mayor disminución en todos los segmentos evaluados $(p<0,05)$. Conclusiones. Las pausas activas supervisadas más folletos informativos lograron disminuir las molestias musculoesqueléticas en trabajadores administrativos. Por ello, puede ser implementado en otras instituciones públicas o privadas.
\end{abstract}

Palabras clave: Dolor musculoesquelético; Programa de salud laboral; Lumbalgia; Dolor de cuello (fuente: DeCS BIREME).

\section{EFFECT OF REST PAUSES COMBINED WITH INFORMATION LEAFLETS ON THE DECREASE IN MUSCULOSKELETAL PAIN IN ADMINISTRATIVE WORKERS}

\begin{abstract}
Objectives. To assess the effect of work pauses combined with information leaflets on the reduction of musculoskeletal pain in administrative workers of a public institution in Lima, Peru. Materials and methods. This quasi-experimental study was conducted in two offices of a public institution and included an intervention group and a non-randomized control group. The intervention group $(n=135)$ was provided with information leaflets once a week, in addition to a program of rest pauses consisting of breathing exercises, stretching, and encouragement. The control group $(n=127)$ received information leaflets once a week. To assess outcomes, the rate and intensity of musculoskeletal pain over the previous 7 days were measured. Results. The mean age of participants was 38 years. The most affected body regions were the neck and lumbar spine. In the intervention group, the rate of musculoskeletal pain was reduced by $20 \%$ in the neck $(p<0.001)$ and $17 \%$ in the lumbar spine $(p<0.001)$ over the previous 7 days. In the control group, the rate of musculoskeletal pain was decreased only in the lumbar spine $(p=0.029)$. The intervention group reported less pain in all evaluated body regions $(p<0.05)$. Conclusions. Rest pauses combined with information leaflets resulted in decreased musculoskeletal pain in administrative workers, indicating that this strategy can be implemented in public and private institutions.
\end{abstract}

Key words: Musculoskeletal pain; Occupational health program; Low back pain; Neck pain (source: MeSH NLM).

\section{INTRODUCCIÓN}

Los trastornos musculoesqueléticos (TME) son las disfunciones más comunes relacionadas al trabajo. Hasta el año 2015, en países de Europa, América Latina e India se han encontrado prevalencias de aproximadamente $50 \%, 60 \%$ y $60 \%$, respectivamente, incluyendo síntomas como dolor, parestesias, hipomovilidad e inflamación (1-5).

\footnotetext{
Escuela de Terapia Física, Universidad Peruana de Ciencias Aplicadas, Lima, Perú.

Dirección de Investigación y Desarrollo, Universidad Científica del Sur, Lima, Perú.

Fisioterapeuta; ${ }^{\mathrm{b}}$ terapeuta ocupacional; ${ }^{\mathrm{c}}$ magíster en Ergonomía; ${ }^{\mathrm{d}}$ magíster en Terapia Manual Ortopédica, ${ }^{\mathrm{e}}$ médico salubrista

Recibido: 27/04/2017 Aprobado: 25/10/2017 En línea: 30/11/2017
}

Citar como: Cáceres-Muñoz VS, Magallanes-Meneses A, Torres-Coronel D, Copara-Moreno P, Escobar-Galindo M, Mayta-Tristán P. Efecto de un programa de pausa activa más folletos informativos en la disminución de molestias musculoesqueléticas en trabajadores administrativos. Rev Peru Med Exp Salud Publica. 2017;34(4):611-8. doi: 10.17843/rpmesp.2017.344.2848 
El desarrollo de molestias musculoesqueléticas (MME) dependerá de los factores de riesgo a nivel físico, organizativo, psicosocial e individual del trabajador (6), es así que existen diferencias en la frecuencia de TME entre el sector administrativo y el sector construcción o agricultura, ya que los factores de riesgo en cada uno de estos sectores presentan características distintas ${ }^{(7)}$. Se conoce, que en los trabajadores administrativos, las zonas más afectadas son el cuello, miembros superiores y la región dorso lumbar, ya que su labor demanda posturas mantenidas, patrones de movimientos rítmicos y repetitivos con pocos periodos de descanso ${ }^{(4,8)}$.

La falta de descanso y los pocos periodos de recuperación aumentan considerablemente el riesgo de sufrir un TME, debido al esfuerzo funcional que se realiza ${ }^{(9)}$. Por lo tanto, un trabajador que permanezca mucho tiempo frente a una computadora sin tener estiramientos adecuados ni pausas activas (PA) que permitan recuperar su equilibrio muscular, o que no reciba un tratamiento adecuado por los síntomas iniciales, estará propenso a desarrollar un TME a futuro ${ }^{(10,11)}$.

Según datos del estudio multinacional Cultural and Psychosocial Influences on Disability (CUPID) que compara países como Costa Rica, Nicaragua y España; la prevalencia de TME en los países con menores ingresos económicos se duplica en comparación a aquellos con mayores ingresos económicos (3). La I Encuesta Centroamericana de Condiciones de Trabajo y Salud considera como válida esta hipótesis, ya que el análisis en los países centroamericanos, confirma los altos índices de TME, sin descartar el factor socioeconómico (2).

En el Perú, la Oficina General de Estadística y Tecnologías de Información y Comunicaciones del Ministerio de Trabajo y Promoción del Empleo, informa que solo fueron notificados cinco casos de TME hasta mayo de 2017, es decir, existe un vacío en la información correspondiente al número real de casos ${ }^{(12)}$. Las posibles causas de la falta de reportes podrían ser el escaso número de médicos que se dedican a realizar dichos diagnósticos ${ }^{(13)}$ o la falta de notificaciones de las organizaciones que tienen la obligación de comunicar sus casos, de acuerdo a la Ley 29783 de Seguridad y Salud en el Trabajo ${ }^{(14)}$.

En el 2014, la oficina de epidemiología del Instituto Nacional de Rehabilitación, informó que el $25,8 \%$ de las deficiencias atendidas en consulta externa fueron de origen musculoesquelético ${ }^{(15)}$. En el mismo año, el Ministerio de Trabajo y Promoción del Empleo notificó que el 30\% de reportes de enfermedades ocupacionales correspondían a TME (16).

En el ámbito de prevención de riesgos en el trabajo, se han ido fomentando estrategias de participación, con la finalidad

\section{MENSAJES CLAVE}

Motivación para realizar elestudio. Las molestias musculoesqueléticas (MME) son frecuentes en trabajadores de oficina y pueden causar ausentismo laboral. Se han desarrollado diferentes enfoques preventivos que incluyen charlas y folletos, pausas activas o desarrollo de actividad física.

Principales hallazgos. Un programa de ocho semanas de duración de pausas activas supervisadas más folletos informativos, dos veces por día, tres veces por semana, tuvo efecto en la disminución de la prevalencia de MME en todos los segmentos corporales menos el cuello. En la intensidad del dolor se tuvo un efecto moderado en todos los segmentos evaluados.

Implicancias. Este programa puede ser implementado para disminuir las MME en trabajadores administrativos.

de mejorar las condiciones laborales y evitar la aparición de TME, disminuyendo así su prevalencia e incidencia ${ }^{(17)}$. De esta manera, también se evita la baja productividad, el ausentismo laboral y las pérdidas económicas (18). En las últimas décadas, el uso de las computadoras ha obligado a los trabajadores administrativos a pasar toda su jornada laboral frente a ellas. Un estudio demostró que el $44 \%$ de estos trabajadores presentaron alguna MME, la mayoría en los segmentos de mano, brazo y cuello, con una incidencia anual de hasta $34 \%$. Del mismo modo, la evidencia epidemiológica confirma que estas molestias están asociadas con las horas de uso de la computadora y a las posturas que adopta el empleado para adaptarse al puesto ${ }^{(19)}$.

Diversos estudios confirman la importancia de los periodos de descanso y de actividad física con entrenamientos de respiración, estiramientos musculares y movilizaciones articulares en diferentes segmentos corporales. Para ello, se han utilizado charlas, softwares o folletos informativos para determinar el efecto de estos frente a la disminución de MME ${ }^{20,21)}$. Además, en otros países se han desarrollado programas de PA, a través de la ejecución de ejercicios de respiración, estiramientos y calentamientos, durante periodos cortos y con una frecuencia variable, que han demostrado una mejoría en la salud ocupacional de los trabajadores ${ }^{(22,23)}$. Sin embargo, si bien en Perú se están implementando programas de PA en diferentes centros laborales, no hemos encontrado estudios que evalúen su efecto sobre las MME.

Por ello, se diseñó un programa basado en PA bajo supervisión y en la entrega de folletos informativos con el objetivo de comprobar el efecto entre ambos grupos y distinguir las diferencias sobre las MME, antes y después de la intervención, para obtener evidencias que permitan su implementación en las instituciones públicas y privadas del Perú. 


\section{MATERIALES Y MÉTODOS}

\section{DISEÑO DE ESTUDIO}

Se realizó un estudio cuasi experimental con grupo control no aleatorizado con medición basal y final, entre noviembre de 2015 y febrero de 2016, con trabajadores del área administrativa de una institución pública en el Perú.

\section{POBLACIÓN Y MUESTRA}

Se incluyeron trabajadores de dos sedes que laboraban en el área administrativa de una institución pública, cada sede cuenta con ocho áreas, de las cuales se eligieron las tres áreas con mayor carga laboral. Cada área presentaba entre 60 y 70 trabajadores. Al grupo intervención o sede A se les brindó folletos informativos más PA supervisadas. Las pausas consistían en ejercicios de respiración, estiramientos musculares y calentamiento, todos bajo supervisión. Al grupo control o sede B se les entregó solo folletos informativos sin supervisión.

Ambos grupos realizan el mismo tipo de trabajo y tienen similares factores de riesgo como mobiliario, ambiente, temperatura e iluminación. Se incluyeron a quienes tuvieran un mínimo de tres meses trabajando en el área, con jornadas de ocho horas diarias de lunes a viernes, y que acepten participar de las intervenciones y mediciones. Se excluyeron a las personas que tenían vacaciones durante el periodo de intervención, gestantes, empleados con antecedentes de cirugía mayor, insuficiencia cardiaca o descanso médico, esta información fue otorgada por el departamento médico de la institución.

Para el cálculo de tamaño de muestra se usó el programa STATA v14.0 y la fórmula de diferencia de proporciones relacionadas, considerando una frecuencia inicial de algún TME ${ }^{(4)}$ y una reducción del $20 \%$ de las frecuencias posintervención, con un nivel de confianza del $95 \%$ y una potencia de $90 \%$, por lo que se requirió que, al menos, 127 sujetos por grupo terminen el estudio.

\section{MEDICIONES}

El desarrollo del estudio tuvo una duración de diez semanas, tiempo recomendado para garantizar la mayor permanencia de los participantes ${ }^{(24)}$. Se realizó una medición de la frecuencia e intensidad de las MME, una semana antes y una semana después de la intervención. Para esto se usó el Cuestionario Nórdico Estandarizado que tiene un coeficiente de alfa de Cronbach de 0,82, y un coeficiente de correlación intraclase de 0,97 ${ }^{(11)}$. Se evaluó la presencia de MME en cuello, hombro, región dorso lumbar, codo/antebrazo y muñeca/mano (positivo o negativo). La intensidad fue evaluada en el mismo periodo y en las mismas áreas, usando una escala de Likert del 0 al $5^{(11)}$.

\section{OTRAS VARIABLES}

Se consideraron otras variables como el tiempo laboral (expresado en años) obtenidos a través de la base de datos de la institución, antecedentes de dolor en los últimos 12 meses por segmento corporal (sí/no), antecedentes de tratamiento para el dolor en los últimos 12 meses por segmento corporal (sí/no), sexo y edad en años.

\section{FOLLETOS INFORMATIVOS}

Los folletos informativos tuvieron como objetivo la educación del trabajador en ergonomía y salud ocupacional. Los temas fueron relacionados a salud ocupacional, ejercicios de respiración, estiramiento muscular, estilos de vida saludable ${ }^{(8)}$ y recomendaciones ergonómicas para el puesto de trabajo. Los folletos fueron entregados por los investigadores una vez por semana durante ocho semanas en ambos grupos.

\section{FOLLETOS INFORMATIVOS MÁS PAUSAS ACTIVAS SUPERVISADAS}

El grupo intervención recibió los folletos informativos además de realizar el programa de PA, el cual fue guiado y supervisado por los investigadores. La intervención fue aplicada durante ocho semanas, con una frecuencia de tres veces por semana, dos veces al día, con turnos de mañana entre las 9 y 10 a.m. y tarde entre las 3 y 4 p.m., la intervención fue aplicada durante su jornada de trabajo. Las pausas activas estuvieron conformadas por ejercicios de respiración, estiramiento muscular y calentamiento. Para ejecutar el programa, los trabajadores suspendían sus actividades laborales de 10 a $15 \mathrm{~min}$. Los ejercicios de respiración consistían en inspiración profunda con posturas de descanso en sedente con los brazos apoyados sobre el escritorio; se realizaron dos series de dos repeticiones por cada PA.

Cada participante realizó estiramientos musculares dirigidos a cuatro segmentos corporales: cuello, miembros superiores, tronco y miembros inferiores. Los estiramientos para cada segmento consistían en la elongación de las fibras musculares durante 10 a $15 \mathrm{~s}$, con una elongación por cada lado. Los ejercicios de calentamiento se basaron en movimientos activos de los segmentos corporales evaluados. Por cada PA se realizó el calentamiento en tres segmentos, uno en zona cervical, otro en miembros superiores $\mathrm{y}$, finalmente, en el tronco, para cada segmento se realizaron diez repeticiones. Estos, a su vez, podían comprender ejercicios posturales y de estabilización articular cervical y/o lumbar. El programa de ejercicios contenía actividades tanto de pie como sentado ${ }^{(5,25,26)}$.

\section{ASPECTOS ÉTICOS}

El estudio fue aprobado por el Comité de Ética de la Universidad Peruana de Ciencias Aplicadas (CEI/682-1015) y por la Jefatura de la institución donde se realizó el 
estudio. Todos los participantes fueron informados de los objetivos del estudio y firmaron el consentimiento informado. Después de terminar el estudio, el grupo que solo recibió folletos también realizo el programa de PA. Además, se capacitó al personal de salud y de recursos humanos de la institución para continuar con este programa.

\section{ANÁLISIS DE DATOS}

Los resultados fueron ingresados a una base de datos en Excel por doble digitación, luego del control fueron exportados al programa STATA v14.0 para el análisis de datos. Las variables categóricas fueron expresadas en frecuencias y porcentajes. El puntaje de intensidad de MME con media y error estándar. Se comparó las frecuencias iniciales y finales entre grupos usando la prueba de chi cuadrado o exacta de Fisher según corresponda. Se comparó el cambio al inicio y al final en cada grupo usando la prueba McNemar. Para evaluar las diferencias en la intensidad de MME al inicio, final y delta (media inicio - media final) se usó la prueba de la suma de rangos Wilcoxon. Finalmente, se calculó el tamaño del efecto de la intensidad del dolor usando la $\mathrm{d}$ de Cohen, $(>0,2$ efecto pequeño; >0,5 efecto moderado; >0,8 efecto grande) ${ }^{(27)}$. Se consideró un valor de $p<0,05$ como significativo.

\section{RESULTADOS}

De 402 personas potencialmente elegibles, 30 tenían algún criterio de exclusión, 372 fueron invitadas a participar del estudio, 60 decidieron no participar y 30 se ausentaron el día de la evaluación basal; finalmente, 20 personas fueron excluidas por llenado incompleto de las variables vinculadas a frecuencia e intensidad. Iniciaron 135 personas en el grupo de intervención y 127 en el grupo control, ninguna abandonó el estudio por lo que todos tuvieron la medición final (Figura 1).

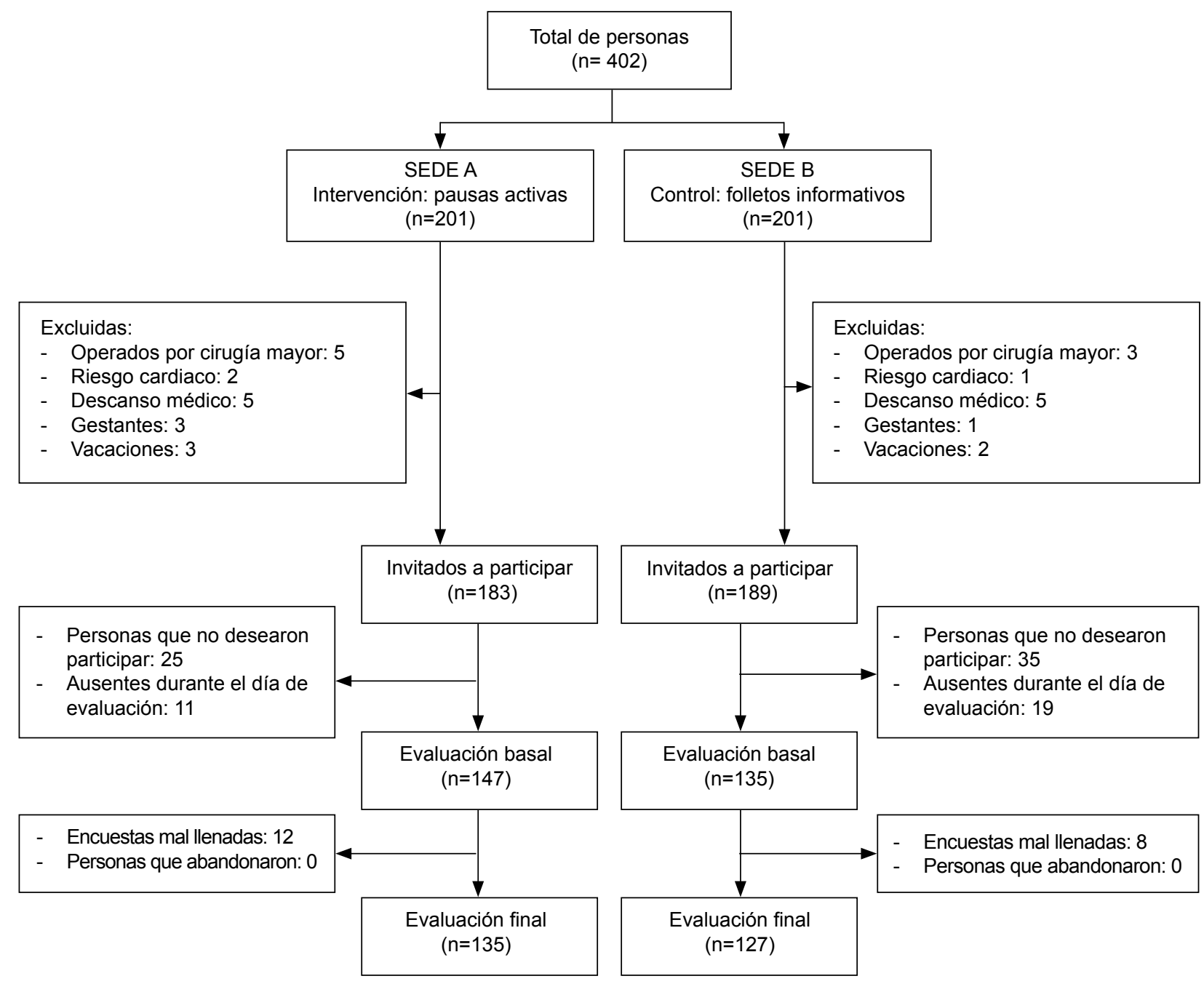

Figura 1. Flujograma de la selección de participantes, según sedes (grupo intervención y grupo control) 
La edad promedio de los participantes fue de 38 años, el $39 \%$ fueron de sexo masculino, la mediana del tiempo laboral fue de 5 años; la zona del cuello y dorso lumbar presentaron mayor prevalencia de MME durante los últimos 12 meses, pero fueron las molestias de hombro y de la región dorso lumbar los que tuvieron mayores antecedentes de tratamientos para el dolor. No se encontraron diferencias en ninguna de las variables medidas entre ambos grupos (Tabla 1).

No se encontró diferencias en la presencia de MME en los últimos siete días en la medición inicial para los segmentos evaluados, excepto en muñeca o mano, donde el grupo control tuvo una mayor frecuencia $(39,4 \%$ vs. $25,2 \% ; p=0,014)$. Asimismo, el grupo de intervención tuvo menores frecuencias respecto al grupo control en todos los segmentos, menos en cuello (Tabla 2).

Los resultados finales del grupo control reflejaron que no se logró reducir la prevalencia en ninguno de sus segmentos $(p>0,05)$ excepto en la zona dorso lumbar $(p=0,029)$; a diferencia del grupo intervención, en donde se redujo en $20 \%$ la frecuencia de molestias de cuello $(p<0,001), 17 \%$ en la zona dorso lumbar $(p<0,001), 7,5 \%$ en hombro $(p=0,025)$ y en $5,9 \%$ en muñeca o mano $(p=0,046)$ (Tabla 3$)$.

Tabla 1. Características de los trabajadores de una institución pública peruana que participaron del estudio.

\begin{tabular}{lrrr}
\hline Características & $\begin{array}{c}\text { Grupo } \\
\text { Intervención } \\
\mathbf{n = 1 3 5}\end{array}$ & $\begin{array}{r}\text { Grupo } \\
\text { Control } \\
\mathbf{n = 1 2 7}\end{array}$ & $\begin{array}{r}\text { Valor } \\
\text { de }^{*}\end{array}$ \\
\hline $\begin{array}{l}\text { Mediana de la edad en } \\
\text { años (RIC) }\end{array}$ & $37(17,0)$ & $38(18)$ & $0,668^{\dagger}$ \\
\hline $\begin{array}{l}\text { Sexo masculino (\%) } \\
\text { Mediana del tiempo laboral } \\
\text { en años (RIC) }\end{array}$ & $58(43,0)$ & $45(35,4)$ & 0,212 \\
\hline Antecedente de dolor (\%) ${ }^{\ddagger}$ & $6(15)$ & $4(9)$ & $0,403^{\dagger}$ \\
\hline Cuello & $104(77,0)$ & $98(77,2)$ & 0,980 \\
\hline $\begin{array}{l}\text { Dorso lumbar } \\
\text { Hombro }\end{array}$ & $86(63,7)$ & $87(68,5)$ & 0,412 \\
\hline Mano / muñeca & $56(41,5)$ & $59(46,5)$ & 0,417 \\
\hline Codo/ antebrazo & $52(38,5)$ & $64(50,4)$ & 0,053 \\
\hline $\begin{array}{l}\text { Antecedente de } \\
\text { tratamiento (\%) }\end{array}$ & $29(21,5)$ & $34(26,8)$ & 0,317 \\
\hline Hombro & & & \\
\hline Dorso lumbar & $20(14,8)$ & $19(15,0)$ & 0,974 \\
\hline Cuello & $19(14,1)$ & $25(19,7)$ & 0,225 \\
\hline Codo/ antebrazo & $11(8,2)$ & $11(8,7)$ & 0,881 \\
\hline Mano / muñeca & $4(3,0)$ & $5(3,9)$ & $0,743 \ddagger$ \\
\hline
\end{tabular}

RIC: Rango intercuartilico

* Prueba de Chi cuadrado

† Suma de rangos de Wilcoxon

‡ Prueba exacta de Fisher

$\S$ En los últimos 12 meses
Tabla 2. Efecto del programa de pausas activas sobre la frecuencia de molestias musculoesqueléticas en los últimos siete días en trabajadores de una institución pública peruana

\begin{tabular}{lccc}
\hline $\begin{array}{l}\text { Molestias } \\
\text { musculoesqueléticas }\end{array}$ & $\begin{array}{c}\text { Grupo } \\
\text { Intervención } \\
\mathbf{n = 1 3 5}\end{array}$ & $\begin{array}{c}\text { Grupo } \\
\text { Control } \\
\mathbf{n = 1 2 7}\end{array}$ & $\begin{array}{c}\text { Valor } \\
\text { de } \mathbf{p}^{*}\end{array}$ \\
\hline Al inicio (\%) & $82(60,7)$ & $71(55,9)$ & 0,427 \\
\hline Cuello & $43(31,9)$ & $43(33,9)$ & 0,730 \\
\hline Hombro & $62(45,9)$ & $64(50,4)$ & 0,469 \\
\hline Dorso lumbar & $19(14,1)$ & $24(18,9)$ & 0,292 \\
\hline Codo/ antebrazo & $34(25,2)$ & $50(39,4)$ & 0,014 \\
\hline Mano / muñeca & & & \\
\hline Al final (\%) & $55(40,7)$ & $65(51,2)$ & 0,090 \\
\hline Cuello & $33(24,4)$ & $48(37,8)$ & 0,019 \\
\hline Hombro & $39(28,9)$ & $55(43,3)$ & 0,015 \\
\hline Dorso lumbar & $13(9,6)$ & $23(18,1)$ & 0,046 \\
\hline Codo/ antebrazo & $26(19,3)$ & $42(33,1)$ & 0,011 \\
\hline Mano / muñeca & & & \\
\hline
\end{tabular}

* Prueba de chi cuadrado

En cuanto a la intensidad de las MME, al inicio no se encontraron diferencias en ninguno de los segmentos, mientras que el grupo intervención tuvo menores puntajes en la medición final $(p<0,05)$ así como al comparar el delta del cambio $(p<0,01)$. Los mayores cambios se dieron en el cuello y en la región dorsal, con un valor de $p<0,001$ en ambas zonas (Tabla 4).

Se verificó que el tamaño del efecto de la PA en el grupo de intervención fue pequeño en las molestias del codo/ antebrazo, moderado en las molestias del hombro y mano/ muñeca y grande en las molestias del cuello y dorso lumbar, tanto en su comparación inicial-final, como al final contra el grupo control (Tabla 5).

Tabla 3. Diferencias en las molestias musculoesqueléticas, entre el inicio y final de la intervención del programa de pausas activas en los últimos siete días en trabajadores de una institución pública peruana

\begin{tabular}{lrrrrr}
\hline Zona & $\begin{array}{c}\text { Grupo } \\
\text { Intervención } \\
\mathbf{n = 1 3 5}\end{array}$ & $\begin{array}{c}\text { Valor } \\
\text { de } \mathbf{p}^{*}\end{array}$ & $\begin{array}{c}\text { Grupo } \\
\text { Control } \\
\mathbf{n = 1 2 7}\end{array}$ & $\begin{array}{l}\text { Valor } \\
\text { de } \mathbf{p}^{*}\end{array}$ \\
\hline Cuello & $27(20,0)$ & $<0,001$ & $6(4,7)$ & 0,201 \\
Hombro & $10(7,5)$ & 0,025 & $-5(-3,9)$ & 0,197 \\
Dorso lumbar & $23(17,0)$ & $<0,001$ & $9(7,1)$ & 0,029 \\
Codo/ & $6(4,5)$ & 0,058 & $1(0,8)$ & 0,739 \\
antebrazo & $8(5,9)$ & 0,046 & $8(6,3)$ & 0,088 \\
\hline Mano / muñeca & $8(\%)$ & & \\
\hline
\end{tabular}

Delta: \%molestias al inicio - \%molestias al final de la intervención

* Prueba de McNemar 
Tabla 4. Efecto del programa de pausas activas sobre la intensidad de molestias musculoesqueléticas en los últimos siete días en trabajadores de una institución pública peruana.

\begin{tabular}{lcccc}
\hline $\begin{array}{l}\text { Molestias } \\
\text { musculoesqueléticas }\end{array}$ & $\begin{array}{c}\text { Grupo } \\
\text { Intervención } \\
\mathbf{n = 1 3 5}\end{array}$ & $\begin{array}{c}\text { Grupo } \\
\text { Control } \\
\mathbf{n = 1 2 7}\end{array}$ & $\begin{array}{c}\text { Valor de } \\
\mathbf{p}^{*}\end{array}$ \\
\cline { 2 - 2 } Media(EE) & Media(EE) & \\
\hline Al inicio & $2,50(0,14)$ & $2,60(0,15)$ & 0,535 \\
\hline Cuello & $1,45(0,16)$ & $1,55(0,16)$ & 0,618 \\
\hline Hombro & $2,20(0,16)$ & $2,40(0,17)$ & 0,365 \\
\hline Dorso lumbar & $0,69(0,12)$ & $0,87(0,14)$ & 0,320 \\
\hline Codo/ antebrazo & $1,23(0,14)$ & $1,57(0,16)$ & 0,090 \\
\hline Mano / muñeca & & & \\
\hline Al final & $0,86(0,09)$ & $2,13(0,15)$ & $<0,001$ \\
\hline Cuello & $0,66(0,09)$ & $1,40(0,15)$ & $<0,001$ \\
\hline Hombro & $0,65(0,08)$ & $1,93(0,16)$ & $<0,001$ \\
\hline Dorso lumbar & $0,26(0,06)$ & $0,72(0,12)$ & 0,018 \\
\hline Codo/ antebrazo & $0,43(0,07)$ & $1,26(0,15)$ & $<0,001$ \\
\hline Mano / muñeca & & & \\
\hline Delta & $1,64(0,10)$ & $0,47(0,11)$ & $<0,001$ \\
\hline Cuello & $0,80(0,12)$ & $0,15(0,11)$ & $<0,001$ \\
\hline Hombro & $1,55(0,12)$ & $0,47(0,11)$ & $<0,001$ \\
\hline Dorso lumbar & $0,43(0,09)$ & $0,15(0,08)$ & 0,008 \\
\hline Codo/ antebrazo & $0,80(0,10)$ & $0,31(0,11)$ & 0,002 \\
\hline Mano / muñeca & & & \\
\hline
\end{tabular}

Delta: media intensidad de molestias al inicio - media intensidad de molestias al final de la intervención, EE: error estándar

* Suma de rangos de Wilcoxon

\section{DISCUSIÓN}

El programa supervisado de PA más folletos informativos logró disminuir la frecuencia e intensidad de las MME en los segmentos estudiados, a excepción del codo/antebrazo.

Los trabajadores evaluados presentan una alta frecuencia de MME así como de intensidad del dolor, particularmente en cuello y en la región dorso lumbar. Similares hallazgos se reportaron en los estudios de Laal $\mathrm{F}$ et al. y Aghilinejad M et al., donde determinaron el grado de MME en sastres y en ensambladores electrónicos (60 y 70\%, respectivamente) al inicio de la intervención, con predominancia de molestias en cuello y en la región dorso lumbar, obteniendo la disminución de la frecuencia posterior a la intervención. Sin embargo, esta intervención ergonómica solo fue en base a charlas y folletos informativos ${ }^{(21,28)}$.

Respecto a los resultados de intensidad de MME, en un inicio, estas fueron moderadas, y luego leves al final de la intervención. Sin embargo, en un estudio similar se hallaron resultados de intensidad moderado, tanto al inicio como al final de la intervención ${ }^{(20)}$. La inconsistencia de este resultado podría deberse a que se realizaron dos PA por día, la primera iniciando la jornada y la siguiente dos horas después.

Las intervenciones con PA deben ir acordes a la demanda de la función muscular o actividad física que realiza el empleado durante sus horas laborales. Se ha demostrado su beneficio en instituciones públicas o centros laborales en donde los empleados suelen mantener posturas durante largos periodos. No obstante, algunos estudios han reportado poco efecto en la reducción de MME, este es el caso de trabajadores de construcción ${ }^{(29)}$ y personal de limpieza ${ }^{(30)}$, debido a que este tipo de población cuenta con factores de riesgo diferentes al de los trabajadores administrativos.

El tiempo establecido para cada sesión de PA fue de 10 a $15 \mathrm{~min}$. Otros estudios realizaron sus intervenciones en rangos similares, de esta manera se refuerza la participación de los trabajadores ${ }^{(31)}$, ya que así no suspenden sus actividades y cumplen con los objetivos laborales programados. Asimismo, un estudio demostró que realizar la intervención con una frecuencia interdiaria, siguiendo una metodología similar a la nuestra, genera mayor participación de los trabajadores en comparación a un programa de intervención diaria ${ }^{(23)}$.

Tabla 5. Tamaño del efecto del programa de pausas activas sobre la intensidad de molestias musculoesqueléticas en los últimos siete días en trabajadores de una institución pública peruana

\begin{tabular}{|c|c|c|c|c|c|c|}
\hline \multirow[t]{2}{*}{ Zona } & \multicolumn{2}{|c|}{$\begin{array}{l}\text { Grupo intervención } \\
\text { Final vs. Inicio }\end{array}$} & \multicolumn{2}{|c|}{$\begin{array}{l}\text { Grupo control } \\
\text { Final vs. Inicio }\end{array}$} & \multicolumn{2}{|c|}{$\begin{array}{c}\text { Final } \\
\text { Intervención } \\
\text { Vs. Control }\end{array}$} \\
\hline & $\begin{array}{c}\text { D de } \\
\text { Cohen }\end{array}$ & IC 95\% & $\begin{array}{l}\text { D de } \\
\text { Cohen }\end{array}$ & IC $95 \%$ & $\begin{array}{c}\text { D de } \\
\text { Cohen }\end{array}$ & IC $95 \%$ \\
\hline Cuello & 1,22 & $0,96-1,48$ & 0,28 & $0,03-0,53$ & 0,94 & $0,68-1,19$ \\
\hline Hombro & 0,52 & $0,28-0,76$ & 0,09 & $-0,16-0,33$ & 0,53 & $0,29-0,78$ \\
\hline Dorso lumbar & 1,07 & $0,82-1,33$ & 0,26 & $0,01-0,50$ & 0,91 & $0,66-1,17$ \\
\hline Codo/ antebrazo & 0,39 & $0,15-0,63$ & 0,10 & $-0,15-0,35$ & 0,42 & $0,18-0,67$ \\
\hline Mano / muñeca & 0,61 & $0,36-0,85$ & 0,18 & $-0,08-0,43$ & 0,65 & $0,40-0,89$ \\
\hline
\end{tabular}

IC 95\%: Intervalos de confianza al 95\% 
Como limitaciones del estudio podemos mencionar que no se realizó aleatorización de las muestras, debido a que esto podría ocasionar un riesgo alto de contaminación (por ello se realizó una intervención distinta en cada sede); el no hacerla afecta teóricamente la comparabilidad. Sin embargo, los trabajadores oficinistas de ambos grupos se desenvuelven en condiciones similares. No se encontró diferencias entre grupos en la medición inicial, es posible que puedan existir diferencias en otras variables no medidas como la obesidad, la actividad física, el estrés o los factores de riesgo psicosocial, que pudieran estar asociadas a las MME ${ }^{(6,31)}$.

Las preguntas del Cuestionario Nórdico Estandarizado que hacen referencia a los segmentos de miembros superiores, cuentan con opciones para que el participante detalle el lado correspondiente al segmento afecto y así determinar si la molestia está en el lado derecho, izquierdo o en ambos. Sin embargo, al referirse al segmento de hombro, la opción "ambos" no se encuentra presente. Por lo tanto, para el análisis se tomó en cuenta como la opción "ambos" a quienes marcaron las dos opciones; izquierdo y derecho, sin realizar cambios en el cuestionario, debido a que este ya se encuentra estructurado y validado de tal forma.

Además, se considera que se tendrían resultados más óptimos si las mediciones de la frecuencia e intensidad de las MME se hubieran realizado semanalmente. Tampoco se tomó en cuenta el cambio del mobiliario, por no estar detallado en el proyecto inicial del estudio.
El programa de PA supervisado, basado en series de ejercicios, logro disminuir el nivel de MME y podría prevenir la aparición de TME. Los resultados obtenidos sugieren que este programa podría ser implementado como parte de la jornada laboral y convertirse en un hábito de salud ocupacional. El efecto de la intervención se demostró con PA realizadas tres veces por semana, dos veces al día. Sin embargo, se requiere de mayor investigación para determinar si estos tiempos son los idóneos para demostrar el efecto de las PA en la disminución de la MME.

El programa de pausas activas supervisadas más folletos informativos logro reducir las molestias musculoesqueléticas en los segmentos de cuello y dorso lumbar en trabajadores oficinistas. Se requiere la realización de estudios aleatorizados, a fin de que este programa puede ser implementado en otras instituciones públicas o privadas de Perú.

Contribuciones de autores: VSCM, AMM, DFC tuvieron la idea del estudio, realizaron la intervención, recogieron los datos, MEG y PCM supervisaron el desarrollo de la intervención, PMT realizó el análisis de los datos, AAM, VSCM y PMT elaboraron el primer borrador del manuscrito. Todos los autores participaron del diseño del estudio, interpretación de los resultados, hicieron contribuciones significativas al manuscrito, aprobaron su versión final y se hacen responsables del contenido.

Fuente de financiamiento: autofinanciado

Conflictos de interés: los autores declaran no tener conflictos de interés con la publicación de este artículo.

\section{REFERENCIAS BIBLIOGRÁFICAS}

1. Eurofound. Primeros resultados: sexta encuentra europea sobre las condiciones de trabajo [Internet]. Dublin: Eurofound Publications Office; 2007. [citado 20 agosto 2017]. Disponible en: https:// www.eurofound.europa.eu/sites/default/ files/ef_publication/field_ef_document/ ef1568es.pdf.

2. Rojas M, Gimeno D, Vargas-Prada S, Benavides FG. Dolor musculoesquelético en trabajadores de América Central: resultados de la I Encuesta Centroamericana de Condiciones de Trabajo y Salud. Rev Panam Salud Publica. 2015;38(2):120-8.

3. Campos-Fumero A, Delclos GL, Douphrate DI, Felknor SA, VargasPrada S, Serra C, et al. Upper extremity musculoskeletal pain amongofficeworkers in three Spanish-speaking countries: findings from the CUPID study. Occup Environ Med. 2016;73(6):394-400. doi: 10.1136/oemed-2015-103327.
4. Kaliniene G, Ustinaviciene R, Skemiene L, Vaiciulis V, Vasilavicius P. Associations between musculoskeletal pain and workrelated factors among public service sector computer workers in Kaunas County, Lithuania. BMC Musculoskeletal Disorders. 2016; 17(1):420. doi 10.1186/s12891-016-1281-7.

5. Pillastrini P, Mugnai R, Bertozzi L, Costi $S$, Curti S, Guccione A, et al. Effectiveness of an ergonomic intervention on workrelated posture and low back pain in video display terminal operators: A 3 year crossover trial. Appl Ergon. 2010;41(3):436 43. doi: 10.1016/j.apergo.2009.09.008.

6. Op De Beeck R, Hermans V. Research on work-related low back disorders [Internet]. Bruselas: Institute for Occupational Safety and Health; 2017 [citado 21 Ago 2017]. Disponible en: https://osha.europa.eu/en/tools-andpublications/publications/reports/204
7. Soe KT, Laosee O, Limsatchapanich S, Rattanapan C. Prevalence and risk factors of musculoskeletal disorders among Myanmar migrant workers in Thai seafood industries. Int J Occup Saf Ergon. 2015;21(4):539-46. doi: 10.1080/10803548.2015.1096609.

8. Shariat A, Mohd Tamrin SB, Arumugam M, Danaee M, Ramasamy R. Office exercise training to reduce and prevent the occurrence of musculoskeletal disorders among office workers: a hypothesis. Malays J Med Sci. 2016;23(4):54-8. doi: 10.21315/mjms2016.23.4.7.

9. KumarS. Theories of musculoskeletal injury causation. Ergonomic. 2001;44(1):17-47.

10. Chaves García M, Martínez D, López Marmolejo A. Evaluación de la carga física postural y su relación con los trastornos musculoesqueléticos. Revista Colombiana de Salud Ocupacional. 2015;4(1):22-25.

11. Kuorinka I, Jonsson B, Kilbom A, Vinterberg H, Biering-Sørensen F, Andersson G 
et al. Standardised Nordic questionnaires for the analysis of musculoskeletal symptoms. Appl Ergon. 1987;18(3):233-7.

12. Boletín estadístico: Notificaciones de accidentes de trabajo, incidentes peligrosos y enfermedades ocupacionales [Internet]. Lima: Ministerio de Trabajo y Promoción del Empleo; 2017 [citado 22 febrero 2017]. Disponible en: http://www2. trabajo.gob.pe/estadisticas/estadisticasaccidentes-de-trabajo/

13. Carrasco V, Lozano E, Velasquez E. Análisis actual y prospectivo de la oferta y demanda de los médicos en el Perú 20052011. Acta Med Per. 2008;25(1):22-29.

14. Ley de Seguridad y Salud en el Trabajo. Ley 29783 de 20 de agosto. El Peruano (20-8-2011). [citado 21 agosto 2017]. Disponible en: http://www.oiss.org/ estrategia/IMG/pdf/01_Ley_29783_de_ Seguridad_y_Salud_en_el_Trabajo_.pdf

15. Sala Situacional: Primer Trimestre-2014. Lima: Oficina de epidemiologia del Instituto Nacional de Rehabilitación; 2014 [Citado 21 agosto 2017]. Disponible en: http://www.inr.gob.pe/transparencia/ Sala\%20Situacional/2014/SALA\%20 SITUACIONAL\%20I\%20TRIMESTRE\%202014.pdf

16. Anuario Estadístico Sectorial de Ministerio de Trabajo y Promoción de Empleo [Internet]. Lima: Ministerio de Trabajo y Promoción de Empleo; 2015 [Citado 21 agosto 2017]. Disponible en: http://www. trabajo.gob.pe/archivos/file/estadisticas/ anuario/ANUARIO_ESTADISTICO_2014.pdf

17. Ruiz-Frutos C, Delclòs J, Ronda E, García AM, Benavides FG. Salud laboral. Conceptos y técnicas para la prevención de riesgos laborales. Barcelona: Elsevier Masson; 2013.

18. Montoya M, Palucci M, Do Carmo R, Taubert F. Lesiones osteomusculares en trabajadores de un hospital mexicano y la ocurrencia del ausentismo. Cienc enferm. 2010;16(2):35-46.
19. IJmker S, Blatter B, van der Beek A, van Mechelen W, Bongers P. Prospective research on musculoskeletal disorders in office workers (PROMO): study protocol. BMC BMC Musculoskelet Disord. 2006;7:55. doi: 10.1186/14712474-7-55.

20. Mehrparvar A, Heydari M, Mirmohammadi S, Mostaghaci M, Davari M, Taheri M. Ergonomic intervention, workplace exercises and musculoskeletal complaints: a comparative study. Med J Islam Repub Iran. 2014;28:69.

21. LaalF, Mirzaei R, Behdani M, Mohammadi M, Khodami K. Evaluation of the influence of ergonomic intervention on the musculoskeletal disorders of Zahedan tailors. Int J Occup Saf Ergon. 2017;23(3):380-385. doi: 10.1080/10803548.2016.1233674.

22. Coffeng JK, Boot CR, Duijts SF, Twisk JW, van Mechelen W, Hendriksen IJ. Effectiveness of a worksite social \& physical environment intervention on need for recovery, physical activity and relaxation; results of a randomized controlled trial. PLoS One. 2014;9(12):e114860. doi: 10.1371/journal.pone.0114860.

23. Andersen LL, Zebis MK. Process evaluation of workplace interventions with physical exercise to reduce musculoskeletal disorders. Int J Rheumatol. 2014;2014:761363. doi: 10.1155/2014/761363.

24. Jakobsen M, Sundstrup E, Brandt M, Jay $\mathrm{K}$, Aagaard P, Andersen L. Physical exercise at the workplace prevents deterioration of work ability among healthcare workers: cluster randomized controlled trial. BMC Public Health. 2015;15:1174. doi: 10.1186/s12889-015-2448-0.

25. Freitas-Swerts FC, Robazzi ML. The effects of compensatory workplace exercises to reduce work-related stress and musculoskeletal pain. Rev Lat Am Enfermagem. 2014;22(4):629-36.
26. Mani K, Provident I, Eckel E. Evidencebased ergonomics education: Promoting risk factor awareness among office computer workers. Work. 2016;55(4): 913-22. doi: 10.3233/WOR-162457.

27. Lakens D. Calculating and reporting effect size to facilitate cumulative science: a practical primer for t-tests and ANOVAs. Front Psychol. 2013;4:863. doi: 10.3389/ fpsyg.2013.00863.

28. Aghilinejada M, Azarb N, Ghasemic M, Dehghana N, Mokamelkhah E. An ergonomic intervention to reduce musculoskeletal discomfort among semiconductor assembly workers. Work. 2016; 54(2):44550. doi: 10.3233/WOR-162325.

29. Jakobsen M, Sundstrup E, Brandt M, Jay K, Aagaard P, Andersen L. Physical exercise at the workplace prevents deterioration of work ability among healthcare workers: cluster randomized controlled trial. BMC Public Health. 2015;15:1174. doi: 10.1186/s12889-015-2448-0.

30. Jorgensen MB, Faber A, Hansen JV, Holtermann A, Sogaard K. Effects on musculoskeletal pain, work ability and sickness absence in a 1-year randomised controlled trial among cleaners. BMC Public Health. 2011;11:840. doi: 10.1186/1471-2458-11-840.

31. Corbeil P, Plamondon A, TeasdaleN,Handrigan G. Biomechanical differences between obese and healthy-weight workers in manual materials handling. Proc Hum Factors Ergon Soc Annu Meet. 2013;57:95861. doi:10.1177/1541931213571213.

\footnotetext{
Correspondencia: Antonella Alejandra Magallanes-Meneses

Dirección: Escuela de Terapia Física, Universidad Peruana de Ciencias Aplicadas, Lima Perú. Av. Alameda San Marcos cuadra 2, Villa (Chorrillos). Lima, Perí.

Teléfono: (+51) 974629458

Correo electrónico: antonellaale71@gmail.com
}

\section{Nuestros artículos se encuentran indizados en:}

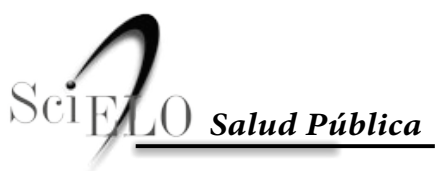

率Www.scielo.org 